\title{
A generalization on the solvability of integral geometry problems along plane curves
}

\section{Zekeriya Ustaoglu*}

\section{"Correspondence:}

zekeriyaustaoglu@karaelmas.edu.tr Department of Mathematics, Bulent Ecevit University, Zonguldak, 67100, Turkey

\begin{abstract}
This paper is concerned with a general condition for the solvability of integral geometry problems along the plane curves of given curvatures. As two important results, the solvabilities of integral geometry problems along the family of circles with fixed radius and along the family of circles of varying radius centered on a fixed circle are given. By using some extension of the class of unknown functions, the proofs are based on the solvabilities of equivalent inverse problems for transport-like equation.
\end{abstract} MSC: 35R30; 53C65; 65N30

Keywords: integral geometry problem; inverse problem; Galerkin method; transport-like equation

\section{Introduction}

The problems of integral geometry are to determine a function, given (weighted) integrals of this function over a family of manifolds, and there has been significant progress in the classical Radon problem when manifolds are hyperplanes and the weight function is unity, there are interesting results in the plane case when a family of curves is regular or in the case of a family of straight lines with arbitrary regular attenuation [1, Chapter 7]. It is assumed that the basis of the integral geometry problems is the Radon transform [2]. The Radon transform $R$ integrates a function $f$ on $\mathbb{R}^{n}$ over hyperplanes. Let $H(s, \Phi)=\left\{x \in \mathbb{R}^{n}\right.$ : $x \cdot \Phi=s\}$ be the hyperplane perpendicular to $\Phi \in S^{n-1}$ (unit sphere) with signed distance $s \in \mathbb{R}^{1}$ from the origin, and the Radon transform $(R f)(s, \Phi)$ is defined as the integral of $f$ over $H(s, \Phi)$, i.e.,

$$
(R f)(s, \Phi)=\int_{H(s, \Phi)} f(x) d x
$$

(see [3, Chapter 2]).

The problems of integral geometry have important applications in imaging and provide the mathematical background of tomography, where the main goal is to recover the interior structure of a nontransparent object using external measurements. The object under investigation is exposed to radiation at different angles, and the radiation parameters are measured at the points of observation. The basic problem in computerized tomography is the reconstruction of a function from its line or plane integrals, and there are many applications related with computerized tomography: medical imaging, geophysics, diagnostic radiology, astronomy, seismology, radar and many other fields (see, e.g., [4]).

(c) 2013 Ustaoglu; licensee Springer. This is an Open Access article distributed under the terms of the Creative Commons Attribution License (http://creativecommons.org/licenses/by/2.0), which permits unrestricted use, distribution, and reproduction in any medium, provided the original work is properly cited. 
From the applied point of view, the importance of integral geometry problem over a family of straight lines in the plane is indicated in [5], where the problem models X-rays, and applicable to the problems of radiology and radiotherapy. Because of their many practical applications, a considerable attention has been devoted to other family of curves in the plane as well as straight lines. Invertibility of the Radon transforms on some families of curves in the plane is given with explicit inversion formulas via circular harmonic decomposition in [6] and for the explicit inversion formulas of the attenuated Radon transform, see, e.g., $[7,8]$. Note that the circle is the simplest non-trivial curve in the plane next to the straight line, and the representation of a function by its circular Radon transform also arises in applications. In [9], invertibility of the Radon transforms over all translations of a circle of fixed radius and circles of varying radius centered on a fixed circle is considered, where the proofs require microlocal analysis of the Radon transforms and a microlocal Holmgren theorem. In [10], some existence and uniqueness results on recovering a function from its circular Radon transform with partial data are presented and the relations to applications in medical imaging are described. There are several other ways related to the selection of a family of curves, such as circles of varying radius centered on a straight line or a fixed curve, circles passing through a fixed point, along paths that are not on the zero sets of harmonic polynomials, circular arcs having a chord of fixed length rotating around its middle point etc., which are meaningful in applications on thermo-acoustic and photoacoustic tomography, synthetic aperture radar, Compton scattering tomography, ultrasound tomography etc. (see, e.g., [10-12] and the references therein).

In fact, since the seminal work of Radon [2], the various integral geometry problems with numerous applications have been considered in several important aspects which are not mentioned here, but for a comprehensive list, see, e.g., [13-16] and the references therein. Furthermore, the problems of integral geometry and inverse problems for transport equations are interrelated and the latter are also of great importance in theory and applications; see, e.g., [17-20] and for the derivation and applications of transport equations, see, e.g., [21-23].

In this paper, a general condition for the solvability of integral geometry problems along plane curves of given curvatures is presented and its relation with some previous results is indicated. Moreover, as two important results, the solvabilities of integral geometry problems along the family of circles with fixed radius and along the family of circles of varying radius centered on a fixed circle are given. Since the curvature of a circle is defined to be the reciprocal of the radius of the circle, in the former case the curvature is a constant, while in the latter one the curvature depends on the point and the direction. To investigate the solvability of the integral geometry problem (IGP) given in Section 2.1, which is overdetermined since the underlying operator of the IGP is compact and its inverse operator is unbounded (see Section 2.2), it is reduced to an equivalent overdetermined inverse problem for a transport-like equation, and then, with the use of a similar method which was proposed in [24] (see also [13, Chapter 1]), on using some extension of the class of unknown functions, this inverse problem is replaced by a determined one. Thus, the solvability of IGP is proved via the solvability of an inverse problem for a transport-like equation. The above mentioned method on the solvability was also previously utilized in [25-27] for IGP along some family of plane curves of given curvatures and straight lines, in [28] for IGP along geodesics and in [29] for IGP along the family of curves whose curvatures are given by the Christoffel symbols. Here, the presented general condition for the 
solvability covers those of [25-28], in the manner indicated by Remark 1 in Section 3.1. Moreover, these previous solvability conditions do not hold for IGP along the above given two families of circles, and this is the main importance and motivation of this study.

In Section 2, IGP and its reduction to the equivalent inverse problem for a transportlike equation, the method to overcome the difficulty on investigating the solvability arising from overdeterminacy of these problems are presented and some definitions and notations which will be used throughout the paper are introduced. Section 3 is devoted to the statements of main results, and finally in Section 4, proofs of the results are given.

The investigation of approximate solutions of the concerned integral geometry problems is beyond the scope of this paper, but similar procedures as in [26] can be carried out by using the Galerkin method or the finite difference method.

\section{Statement of the problem and overdeterminacy}

\subsection{Statement of the problem}

Let $D$ be a bounded domain in $\mathbb{R}^{2}$. It is assumed that in $D$, a family of regular curves is given by curvature $K(x, \varphi)$ which is the curvature of the curve passing from the point $x=$ $\left(x_{1}, x_{2}\right) \in D$ in the direction $v=(\cos \varphi, \sin \varphi)$, and there exists a unique sufficiently smooth curve of this family which is passing from any point $x \in D$ in the arbitrary direction $v$, with the endpoints on the boundary of $D$. Suppose that the lengths of these curves in $D$ are bounded above by the same constant. Let us denote the family of these curves by $\{\Gamma\}$. IGP is stated below.

IGP Determine a function $\lambda(x)$ in the domain $D$ from the integrals of $\lambda$ along the curves of a given family of curves $\{\Gamma\}$.

Suppose that $\lambda(x) \in C\left(\mathbb{R}^{2}\right)$ vanishes outside $D$, and let us introduce an auxiliary function

$$
u(x, \varphi)=\int_{\gamma(x, \varphi)} \lambda d \sigma
$$

where $\gamma(x, \varphi)$ is a part of the curve that belongs to $\{\Gamma\}$, with one end of it being the point $x$ and the other one on $\partial D$, and $d \sigma$ is the arc length element along $\gamma(x, \varphi)$.

Investigating the uniqueness of a solution of a problem of integral geometry by reducing it to the equivalent inverse problem for a differential equation was first carried out in [30]. Similar reduction is demonstrated for IGP formulated below.

Differentiating (1) in the direction $v$ at $x$, we obtain the following transport-like equation:

$$
L u \equiv u_{x_{1}} \cos \varphi+u_{x_{2}} \sin \varphi+K(x, \varphi) u_{\varphi}=\lambda(x) .
$$

From (1), $u$ is $2 \pi$-periodic with respect to $\varphi$, and since the integrals of $\lambda$ along the curves of $\{\Gamma\}$ are known, $u$ is known on $\partial D \times(0,2 \pi)$, i.e.,

$$
\left.u\right|_{\partial D \times(0,2 \pi)}=u_{0}(x, \varphi), \quad u(x, \varphi)=u(x, \varphi+2 \pi)
$$

(see [30] and [13, p.11]). So, we have the following inverse problem.

Problem 1 Determine a pair of functions $(u, \lambda)$ from the transport-like equation (2) provided that the function $K$ is known and $u$ satisfies conditions (3). 


\subsection{Overdeterminacy}

Generally, in the theory of integral geometry, reconstruction of a function of $n$ variables from a function of $m>n$ variables is said to be an overdetermined problem of integral geometry (see, e.g., [14, Chapter 5]), and in the theory of inverse problems, overdetermination usually means that the number $m$ of independent variables in the data exceeds the number $n$ of independent variables in the unknown target function (see, e.g., [31, Section 1.3]). However, since the data $u_{0}$ given on the two-dimensional surface $\partial D \times(0,2 \pi)$ and $\lambda(x)$ is a function of two variables, these are not the cases for IGP or Problem 1. Here, since the operator given in (1) (for $\gamma(x, \varphi)$, where $(x, \varphi) \in \partial D \times(0,2 \pi)$ ) is compact, its inverse is unbounded, and therefore it is not possible to prove a general existence result. So, IGP and Problem 1 are called overdetermined in this sense. Hence, because of the overdeterminacy, the initial data for these problems should not be arbitrary and satisfy some 'solvability conditions' (see [13, p.4] and [15, p.18, Theorem 1.4]) which are difficult to establish. It should be noted that the set of functions $u_{0}$ for which IGP is solvable is not everywhere dense in any of the spaces $L_{2}(\partial D \times(0,2 \pi)), C^{m}(\partial D \times(0,2 \pi))$ and $H^{m}(\partial D \times(0,2 \pi))$. Moreover, the data in problems of integral geometry are of quasianalytic character, i.e., their values specified in a domain of the Lebesgue measure can be as small as desired, determine their values in an essentially larger domain (see [32, Chapter 6, Section 17] and [33, Chapter 6, Section 1]). In particular, this implies that it is impossible to avoid overdeterminacy of the problem by specifying the data on a part of the boundary rather than on the whole boundary. Even if it were possible to find the solvability conditions for the mentioned overdetermined problems, since the real data usually have some errors in practice, and thus fall out of the data class for which the existence of a solution is established, it appears that these conditions would not always be satisfactory in applications. Therefore, to prove the existence results, such special conditions on the data $u_{0}$ have to be posed.

Let us propose the procedure for establishing the solvability of IGP. Assume that the unknown function $\lambda$ in IGP depends not only upon the space variables $x$, but also upon the direction $\varphi$ in some special manner, i.e., consider $\lambda(x, \varphi)$, where this dependence upon $\varphi$ is impossible to be arbitrary, for in the opposite case the problem would be underdetermined and the examples on the nonuniqueness of a solution can be easily constructed. Herein the special dependence of $\lambda(x, \varphi)$ upon the direction means that $\lambda(x, \varphi)$ satisfies a certain differential equation ( $\hat{L} \lambda=0$, where the expression of $\hat{L}$ is given in Section 2.3) with the following properties:

(1) The IGP or Problem 1 with the function $\lambda(x, \varphi)$ becomes a determined one.

(2) The sufficiently smooth functions $\lambda$ depending only on $x$ satisfy this equation.

Suppose that a differential equation for $\lambda(x, \varphi)$ satisfying properties (1) and (2) has been found and that a priori the function $u_{0}^{e}$, which represents the exact data of IGP related to a function $\lambda$ depending only on $x$, is known. Then, utilizing $u_{0}^{e}$, a solution $\tilde{\lambda}$ to IGP can be constructed. By uniqueness of a solution, $\tilde{\lambda}$ and $\lambda(x)$ coincide. At the same time, knowing the approximate data $u_{0}^{a}$ with $\left\|u_{0}^{e}-u_{0}^{a}\right\|_{H^{3}(\partial D \times(0,2 \pi))} \leq \varepsilon$, an approximate solution $\lambda^{a}(x, \varphi)$ can be constructed such that $\left\|\lambda-\lambda^{a}\right\|_{L_{2}(\Omega)} \leq \varepsilon C$. Recall that if $\lambda$ depends only on $x$ and $u_{0}^{a}$ does not satisfy the 'solvability conditions', the solution $\lambda^{a}$ depending only $x$ does not exist. Here the data are specified on $\partial D \times(0,2 \pi)$ and $C>0$ is independent of $u_{0}^{e}$ and $u_{0}^{a}$. In other words, a regularizing procedure is constructed for the IGP. 
In general, the equation with the properties (1) and (2) for the same problem is not uniquely defined. Hence, the class of unknown functions $\lambda$ extends so that IGP for this class becomes a determined problem and all sufficiently smooth functions in $x$ belong to it. On using some extension of the class of functions $\lambda$, the overdetermined Problem 1 is replaced by a determined one (Problem 2 in Section 3.1).

The above method of solvability of the IGP or Problem 1 leads to the Dirichlet-type problem with conditions (3) for the third-order equation of the form $A u \equiv \hat{L} L u=\mathcal{F}$. In investigating and proving the solvability of IGP over any regular family $\{\Gamma\}$ of curves with curvature $K$, since the quadratic form $J(\nabla u)$ in (14) is required to be positive definite, the construction of $\hat{L}$ is important and to be able to this, in Theorem 1 condition (6) is given.

\subsection{Definitions and notations}

In this section, some notations are given based on [13]. Let $D \subset \mathbb{R}^{2}$ with the boundary $\partial D \in C^{3}, \Omega=\{(x, \varphi): x \in D, \varphi \in(0,2 \pi)\}$ and $\bar{\Omega}$ be the closure of $\Omega$. By $(u, v)_{L_{2}(\Omega)}$ we denote a scalar product of functions $u$ and $v$ in $L_{2}(\Omega)$ and by $C_{0}^{\infty}(\Omega)$ the set of all functions defined in $\Omega$ which have continuous partial derivatives of order up to all $k<\infty$, whose supports are compact subsets of $\Omega$. For a differential expression $A$, by $A^{*}$ we denote the conjugate of $A$ in the sense of Lagrange. For $x=\left(x_{1}, x_{2}\right)$,

$$
\begin{aligned}
& \partial_{x_{i}} u=\frac{\partial u}{\partial x_{i}}=u_{x_{i}} \quad(i=1,2), \quad \partial_{\varphi} u=\frac{\partial u}{\partial \varphi}=u_{\varphi} \\
& \left|\nabla_{x} u\right|^{2}=u_{x_{1}}^{2}+u_{x_{2}}^{2} \quad \text { and } \quad\left|\nabla_{x, \varphi} u\right|^{2}=\left|\nabla_{x} u\right|^{2}+u_{\varphi}^{2} .
\end{aligned}
$$

Let $C_{\pi}^{3}(\Omega)$ denote the set of real-valued functions $u \in C^{3}(\Omega)$ that are $2 \pi$-periodic with respect to $\varphi$ in the domain $\Omega$, i.e., $\partial_{x_{1}}^{\alpha_{1}} \partial_{x_{2}}^{\alpha_{2}} \partial_{\varphi}^{\alpha_{3}} u(x, 0)=\partial_{x_{1}}^{\alpha_{1}} \partial_{x_{2}}^{\alpha_{2}} \partial_{\varphi}^{\alpha_{3}} u(x, 2 \pi)$, where $\alpha_{i}$ are nonnegative integers such that $0 \leq \alpha_{1}+\alpha_{2}+\alpha_{3} \leq 3$.

The proof of Theorem 1 involves energy-like estimates and the Galerkin method (see, e.g., [34, Chapter 5, Section 2.3], [35, Chapter 7]), and therefore some class of functions are introduced below. In $C_{\pi}^{3}(\Omega)$, let us introduce the scalar product

$$
(u, v)_{1,2}=\int_{\Omega}\left(u v+\sum_{i=1}^{2}\left(u_{x_{i}} v_{x_{i}}+u_{x_{i} \varphi} v_{x_{i} \varphi}\right)+u_{\varphi} v_{\varphi}+u_{\varphi \varphi} v_{\varphi \varphi}\right) d \Omega,
$$

where $d \Omega=d x_{1} d x_{2} d \varphi$ and set $\|u\|_{1,2}=\left[(u, u)_{1,2}\right]^{1 / 2}$. Let $H_{1,2}^{\pi}(\Omega)$ and $H_{m}^{\pi}(\Omega)$ be the completions of $C_{\pi}^{3}(\Omega)$ with respect to the norms $\|\cdot\|_{1,2}$ and $\|\cdot\|_{H^{m}(\Omega)}(m=1,2,3)$, respectively (for the space $H^{m}$, see, e.g., $[34,36]$ ).

Let $C_{\pi 0}^{3}=\left\{w:\left.w\right|_{\partial D \times(0,2 \pi)}=0, w \in C_{\pi}^{3}(\Omega)\right\}$, and $\stackrel{\circ}{1,2}_{1}^{\pi}(\Omega)$ and $\stackrel{\circ}{m}_{m}^{\pi}(\Omega)$ be the completions of $C_{\pi 0}^{3}$ with respect to the norms $\|\cdot\|_{1,2}$ and $\|\cdot\|_{H^{m}(\Omega)}(m=1,2,3)$. Let us take a set $\left\{w_{1}, w_{2}, \ldots\right\} \subset C_{\pi 0}^{3}$ which is complete and orthonormal in $L_{2}(\Omega)$, then we may assume that the linear span of this set is everywhere dense in $\stackrel{\circ}{1,2}^{\pi}(\Omega)$. Since $\stackrel{\circ}{1,2}^{\pi}(\Omega) \cap \stackrel{\circ}{H}_{1}(\Omega)$ is separable, there exists a countable set $\left\{\varphi_{i}\right\}_{i=1}^{\infty} \subset C_{\pi 0}^{3}$ which is everywhere dense in this space and this set up can be extended to a set which is everywhere dense in $L_{2}(\Omega)$. Orthonormalizing the latter in $L_{2}(\Omega)$, we obtain $\left\{w_{1}, w_{2}, \ldots\right\}$. We denote by $\mathcal{P}_{n}$ the orthogonal projector of $L_{2}(\Omega)$ onto $\mathcal{M}_{n}$ which is the linear span of $\left\{w_{1}, w_{2}, \ldots, w_{n}\right\}$. 
Let

$$
\begin{aligned}
\hat{L} u & =\frac{\partial}{\partial l}\left(\frac{\partial}{\partial \varphi} u\right), \\
\frac{\partial}{\partial l} & =(\sin \varphi) \frac{\partial}{\partial x_{1}}-(\cos \varphi) \frac{\partial}{\partial x_{2}}+g \frac{\partial}{\partial \varphi}+g_{\varphi},
\end{aligned}
$$

where $g(x, \varphi) \in C_{\pi}^{1}(\Omega)$ and it can be easily verified that

$$
\left(\frac{\partial}{\partial l}\right)^{*}=-(\sin \varphi) \frac{\partial}{\partial x_{1}}+(\cos \varphi) \frac{\partial}{\partial x_{2}}-g \frac{\partial}{\partial \varphi} .
$$

The existence of the function $g$ in the expression of $\frac{\partial}{\partial l}$ leads to a generalization on conditions for the solvability of integral geometry problems. In Theorem 1 , it is shown that if there exists a function $g$ satisfying condition (6) which depends on the curvature $K$ and the domain $D$, the solvability holds.

Let $A u \equiv \hat{L} L u$ and $\Gamma^{\prime \prime}(A)$ be the set of all functions $u \in L_{2}(\Omega)$ such that for any $u \in$ $\Gamma^{\prime \prime}(A)$ there exists $y \in L_{2}(\Omega)$ such that $A u=y$ in the generalized functions sense, i.e., $\left(u, A^{*} \eta\right)_{L_{2}(\Omega)}=(y, \eta)_{L_{2}(\Omega)}$ holds for every $\eta \in C_{0}^{\infty}(\Omega)$. Take a subset $\Gamma(A) \subset \Gamma^{\prime \prime}(A)$ such that for any $u \in \Gamma(A)$ there exists a sequence $\left\{u_{k}\right\} \subset C_{\pi 0}^{3}$ such that $u_{k} \rightarrow u$ weakly in $L_{2}(\Omega)$ and $\left(A u_{k}, u_{k}\right)_{L_{2}(\Omega)} \rightarrow(A u, u)_{L_{2}(\Omega)}$ as $k \rightarrow \infty$. If we denote the closure of $C_{\pi 0}^{3}$ with respect to the norm $\|u\|_{\Gamma(A)}=\|u\|_{L_{2}(\Omega)}+\|A u\|_{L_{2}(\Omega)}$ by $\Gamma^{\prime}(A)$, then we have $\Gamma^{\prime}(A) \subset \Gamma(A) \subset \Gamma^{\prime \prime}(A)$ and it can be shown that the inclusions $\stackrel{\circ}{H}_{3}^{\pi}(\Omega) \subset \Gamma^{\prime \prime}(A) \cap \stackrel{\circ}{H}_{1,2}^{\pi}(\Omega) \subset \Gamma(A) \subset L_{2}(\Omega)$ hold.

\section{Statements of results}

\subsection{Solvability of IGP along plane curves}

Since Problem 1 is overdetermined, as indicated in Section 2.2, we consider the following determined problem.

Problem 2 Determine a pair of functions $(u, \lambda)$ defined in $\Omega$ that satisfies

$$
L u=\lambda(x, \varphi)
$$

provided that $\hat{L} \lambda=0, u$ is $2 \pi$-periodic with respect to $\varphi,\left.u\right|_{\partial D \times(0,2 \pi)}=u_{0}$ and $K$ are known.

In (4), it is assumed that the unknown function $\lambda$ depends also on $\varphi$ and the condition $\hat{L} \lambda=0$ holds in the generalized functions sense.

If $u_{0} \in C^{3}(\partial D \times(0,2 \pi))$ and $\partial D \in C^{3}$, then there exists a function $G \in C_{\pi}^{3}(\bar{\Omega})$ (see [34, p.130, Theorem 2]) such that $\left.G\right|_{\partial D \times(0,2 \pi)}=u_{0}$, and we can consider the new unknown function $\bar{u}=u-G$. Hence from (4) we obtain the equation $L \bar{u}=\lambda+F$, where $F=-L G$ and $\left.\bar{u}\right|_{\partial D \times(0,2 \pi)}=0$. Let us denote $\bar{u}$ again by $u$ for simplicity, then Problem 2 can be reduced to Problem 3 given below (see [13, p.20]) and the solvability of the former follows from that of the latter and does not depend on the choice of $G$.

Problem 3 Determine a pair of functions $(u, \lambda)$ defined in $\Omega$ that satisfies

$$
L u=\lambda(x, \varphi)+F,
$$


provided that $\hat{L} \lambda=0, u$ is $2 \pi$-periodic with respect to $\varphi,\left.u\right|_{\partial D \times(0,2 \pi)}=0, K$ and $F$ are known.

The existence, uniqueness and the stability of the solution of Problem 3 are given by the following theorem.

Theorem 1 If $F \in H_{2}^{\pi}(\Omega)$ and a function $g(x, \varphi) \in C_{\pi}^{1}(\Omega)$ exists such that

$$
g_{x_{1}} \cos \varphi+g_{x_{2}} \sin \varphi+g_{\varphi} K-K_{x_{1}} \sin \varphi+K_{x_{2}} \cos \varphi-K_{\varphi} g>K^{2}+g^{2},
$$

for all $(x, \varphi) \in \bar{D} \times(0,2 \pi)$, then Problem 3 has a unique solution $(u, \lambda)$ such that $u \in \Gamma(A) \cap$ $\stackrel{\circ}{H}_{1}^{\pi}(\Omega), \lambda \in L_{2}(\Omega)$ and

$$
\|u\|_{\hat{H}_{1}^{\pi}(\Omega)}+\|\lambda\|_{L_{2}(\Omega)} \leq C\left(\|F\|_{L_{2}(\Omega)}+\left\|F_{\varphi}\right\|_{L_{2}(\Omega)}\right)
$$

holds, where $C>0$ depends on $K$ and the Lebesgue measure of $D$.

Remark 1 In fact, without being aware of (6), the function $g$, with the appropriate choices of it, was used previously in $[25,26,28]$. The convenience of (6) with those of previous solvability results is indicated below.

(i) In [26], when the curvature $K$ is sufficiently smooth and $2 \pi$-periodic, the condition for the solvability is $-(\sin \varphi) \frac{\partial K}{\partial x_{1}}+(\cos \varphi) \frac{\partial K}{\partial x_{2}}>K^{2}$ for all $(x, \varphi) \in \bar{D} \times(0,2 \pi)$, where this condition holds for $g=0$ in (6).

(ii) In [25], when $K(x, \varphi)=f_{2}(x) \cos \varphi-f_{1}(x) \sin \varphi$, where $f_{1}(x)$ and $f_{2}(x)$ are sufficiently smooth functions, the condition for the solvability is $\frac{\partial f_{1}}{\partial x_{1}}+\frac{\partial f_{2}}{\partial x_{2}}>0$ for all $x \in \bar{D}$, which holds for $g(x, \varphi)=f_{1}(x) \cos \varphi+f_{2}(x) \sin \varphi$ in (6).

(iii) In [28], when $K(x, \varphi)=f_{1}(x, \varphi) \cos \varphi+f_{2}(x, \varphi) \sin \varphi$, where $f_{1}(x, \varphi)$ and $f_{2}(x, \varphi)$ are sufficiently smooth $2 \pi$-periodic functions, the condition for the solvability is $\frac{\partial f_{1}}{\partial x_{2}}-\frac{\partial f_{2}}{\partial x_{1}}+f_{2} \frac{\partial f_{1}}{\partial \varphi}-f_{1} \frac{\partial f_{2}}{\partial \varphi}>0$ for all $(x, \varphi) \in \bar{D} \times(0,2 \pi)$, and this condition holds for $g(x, \varphi)=f_{1}(x, \varphi) \sin \varphi-f_{2}(x, \varphi) \cos \varphi$ in (6).

Note that in none of the above cases, the solvability conditions hold for the IGP along the family of circles of varying radius centered on a fixed circle and the family of curves of constant curvatures, i.e., the family of circles with fixed radius where the curvature is a nonzero constant or the family of straight lines where the curvature is zero. The former cases are investigated in Section 3.2 below and the latter case is considered in [27], where the term $K u_{\varphi}$ in the expression of $L u$ will not be present since $K=0$ and the proof of solvability is given for $g=0$. In fact, the strict inequality in (6) can be written as a nonstrict inequality, with the equality only for $K=g=0$.

\subsection{Solvability of IGP along the family of circles}

Since Theorem 1 was given for IGP along a regular family of plane curves for the general case, in this section the given results on the solvabilities of IGP along the family of circles depend on finding an appropriate function $g$ satisfying (6) for the given curvature $K$ and the domain $D$ under the assumptions of Theorem 1 . 


\subsubsection{Solvability of IGP along the family of circles with fixed radius}

Let $D \subset \mathbb{R}^{2}$ and $M=\sup _{\left(x_{1}, x_{2}\right) \in D}\left(x_{1}^{2}+x_{2}^{2}\right)$. Let us take the family of curves in IGP as the family of circular arcs (the segments of circles inside $D$ with the endpoints on $\partial D$ ) with fixed radius $r$, passing from the point $\left(x_{1}, x_{2}\right) \in D$ in the direction $v=(\cos \varphi, \sin \varphi)$ and denote this family of circles by $\left\{\Gamma_{r}\right\}$. Since the curvature of a circle is defined to be the reciprocal of the radius of this circle, the curvature of the elements of the family $\left\{\Gamma_{r}\right\}$ becomes $K=\frac{1}{r}$.

The solvability of IGP along the curves of $\left\{\Gamma_{r}\right\}$ follows from the following lemma.

Lemma 1 Let us define $g$ on $D \times(0,2 \pi)$ as

$$
g\left(x_{1}, x_{2}, \varphi\right)=\frac{1}{3 M}\left(x_{1} \cos \varphi+x_{2} \sin \varphi\right) .
$$

If $r>3 \sqrt{M}$, then (6) holds for $K=\frac{1}{r}$.

Remark 2 The above choice of $g$ is not unique, and since the curvature $K=\frac{1}{r}$ is constant, and hence $K_{x_{1}}=K_{x_{2}}=K_{\varphi}=0$, condition (6) reduces to

$$
g_{x_{1}} \cos \varphi+g_{x_{2}} \sin \varphi+g_{\varphi} \frac{1}{r}>\frac{1}{r^{2}}+g^{2}
$$

for all $(x, \varphi) \in \bar{D} \times(0,2 \pi)$, which depends on $r$ and the domain $D$.

\subsubsection{Solvability of IGP along the family of circles of varying radius centered on a fixed} circle

Let $D \subset \mathbb{R}^{2}$ and $M=\sup _{\left(x_{1}, x_{2}\right) \in D}\left(x_{1}^{2}+x_{2}^{2}\right)$. Let $D_{R}$ be a fixed disk of radius $R>0$ and, without any loss of generality, centered at the origin such that $R>\sqrt{M}$, and take the family of curves in IGP as the family of circular arcs (the segments of circles inside $D$ with the endpoints on $\partial D$ ) of varying radius $r\left(x_{1}, x_{2}, \varphi\right)$ centered on $\partial D_{R}$, passing from the point $\left(x_{1}, x_{2}\right) \in D$ in the direction $v=(\cos \varphi, \sin \varphi)$ and denote this family of circular arcs by $\left\{\Gamma_{R}\right\}$. It can be shown that the radius of the circles of the family $\left\{\Gamma_{R}\right\}$ is defined by the function

$$
r\left(x_{1}, x_{2}, \varphi\right)=x_{1} \sin \varphi-x_{2} \cos \varphi+\left(R^{2}-\left(x_{1} \cos \varphi+x_{2} \sin \varphi\right)^{2}\right)^{1 / 2},
$$

and hence the curvature of the elements of the family $\left\{\Gamma_{R}\right\}$ is defined by

$$
K\left(x_{1}, x_{2}, \varphi\right)=\left(x_{1} \sin \varphi-x_{2} \cos \varphi+\left(R^{2}-\left(x_{1} \cos \varphi+x_{2} \sin \varphi\right)^{2}\right)^{1 / 2}\right)^{-1}
$$

on $D \times(0,2 \pi)$. Note that since $R>\sqrt{M}$, we have $r\left(x_{1}, x_{2}, \varphi\right)>0$ and $K\left(x_{1}, x_{2}, \varphi\right)>0$ on $D \times(0,2 \pi)$.

The solvability of IGP along the curves of $\left\{\Gamma_{R}\right\}$ follows from Lemma 2 given below.

Lemma 2 If the function $g$ is defined on $D \times(0,2 \pi)$ as

$$
g\left(x_{1}, x_{2}, \varphi\right)=\frac{1}{2 M}\left(x_{1} \cos \varphi+x_{2} \sin \varphi\right)
$$

then (6) holds for $K$ defined in (11). 


\section{Proof of results}

Proof of Theorem 1 First, we will prove the uniqueness of the solution $(u, \lambda)$ of Problem 3 under the assumptions of the theorem. To this end, it is sufficient to show that the corresponding homogeneous problem has only a trivial solution. Then, by taking into account that $\hat{L} \lambda=0$ and $F=0$, from (5) we obtain $\hat{L} L u=A u=0$.

Since $u \in \Gamma(A)$, there exists a sequence $\left\{u_{k}\right\} \subset C_{\pi 0}^{3}$ such that $u_{k} \rightarrow u$ weakly in $L_{2}(\Omega)$ and $\left(A u_{k}, u_{k}\right)_{L_{2}(\Omega)} \rightarrow(A u, u)_{L_{2}(\Omega)}=0$ as $k \rightarrow \infty$. Now we want to decompose the product $\left(A u_{k}\right) u_{k}$ into the sum of a positive definite quadratic form and a divergence form. For this purpose, we have the following identities:

$$
\begin{aligned}
\left(A u_{k}\right) u_{k}= & \left(\hat{L} L u_{k}\right) u_{k} \\
= & \left(\frac{\partial}{\partial l}\left(\frac{\partial}{\partial \varphi} L u_{k}\right)\right) u_{k} \\
= & \frac{\partial}{\partial \varphi} L u_{k}\left(\frac{\partial}{\partial l}\right)^{*} u_{k}+\frac{\partial}{\partial x_{1}}\left(u_{k}\left(\frac{\partial}{\partial \varphi} L u_{k}\right) \sin \varphi\right) \\
& -\frac{\partial}{\partial x_{2}}\left(u_{k}\left(\frac{\partial}{\partial \varphi} L u_{k}\right) \cos \varphi\right)+\frac{\partial}{\partial \varphi}\left(u_{k}\left(\frac{\partial}{\partial \varphi} L u_{k}\right) g\right)
\end{aligned}
$$

and

$$
\begin{aligned}
2 \frac{\partial}{\partial \varphi} L u_{k}\left(\frac{\partial}{\partial l}\right)^{*} u_{k}= & 2\left(u_{k x_{1} \varphi} \cos \varphi-u_{k x_{1}} \sin \varphi+u_{k x_{2} \varphi} \sin \varphi+u_{k x_{2}} \cos \varphi\right. \\
& \left.+K_{\varphi} u_{k \varphi}+K u_{k \varphi \varphi}\right)\left(-u_{k x_{1}} \sin \varphi+u_{k x_{2}} \cos \varphi-g u_{k \varphi}\right) \\
= & u_{k x_{1}}^{2}+u_{k x_{2}}^{2}+2 K u_{k \varphi}\left(u_{k x_{1}} \cos \varphi+u_{k x_{2}} \sin \varphi\right) \\
& +2 g u_{k \varphi}\left(u_{k x_{1}} \sin \varphi-u_{k x_{2}} \cos \varphi\right)+\left(g_{x_{1}} \cos \varphi+g_{x_{2}} \sin \varphi\right. \\
& \left.+g_{\varphi} K-K_{x_{1}} \sin \varphi+K_{x_{2}} \cos \varphi-g K_{\varphi}\right) u_{k \varphi}^{2} \\
& +\frac{\partial}{\partial x_{1}}\left(u_{k x_{2}} u_{k \varphi}+K u_{k \varphi}^{2} \sin \varphi-g u_{k \varphi}^{2} \cos \varphi\right) \\
& -\frac{\partial}{\partial x_{2}}\left(u_{k x_{1}} u_{k \varphi}+K u_{k \varphi}^{2} \cos \varphi+g u_{k \varphi}^{2} \sin \varphi\right) \\
& +\frac{\partial}{\partial \varphi}\left(-u_{k x_{1}}^{2} \sin \varphi \cos \varphi+u_{k x_{2}}^{2} \sin \varphi \cos \varphi+u_{k x_{1}} u_{k x_{2}} \cos 2 \varphi\right. \\
& \left.-2 K u_{k x_{1}} u_{k \varphi} \sin \varphi+2 K u_{k x_{2}} u_{k \varphi} \cos \varphi-g K u_{k \varphi}^{2}\right) .
\end{aligned}
$$

If (6) holds, then the quadratic form $J\left(\nabla u_{k}\right)$ in $u_{k x_{1}}, u_{k x_{2}}, u_{k \varphi}$ is positive definite, where

$$
\begin{aligned}
J\left(\nabla u_{k}\right)= & u_{k x_{1}}^{2}+u_{k x_{2}}^{2}+2 K u_{k \varphi}\left(u_{k x_{1}} \cos \varphi+u_{k x_{2}} \sin \varphi\right) \\
& +2 g u_{k \varphi}\left(u_{k x_{1}} \sin \varphi-u_{k x_{2}} \cos \varphi\right)+\left(g_{x_{1}} \cos \varphi+g_{x_{2}} \sin \varphi\right. \\
& \left.+g_{\varphi} K-K_{x_{1}} \sin \varphi+K_{x_{2}} \cos \varphi-g K_{\varphi}\right) u_{k \varphi}^{2} \\
= & u_{k x_{1}}^{2}+u_{k x_{2}}^{2}+2 u_{k \varphi} u_{k x_{1}}(K \cos \varphi+g \sin \varphi) \\
& +2 u_{k \varphi} u_{k x_{2}}(K \sin \varphi-g \cos \varphi)+\left(g_{x_{1}} \cos \varphi+g_{x_{2}} \sin \varphi\right. \\
& \left.+g_{\varphi} K-K_{x_{1}} \sin \varphi+K_{x_{2}} \cos \varphi-g K_{\varphi}\right) u_{k \varphi}^{2} .
\end{aligned}
$$


Indeed, we can estimate the terms $2 u_{k \varphi} u_{k x_{1}}(K \cos \varphi+g \sin \varphi)$ and $2 u_{k \varphi} u_{k x_{2}}(K \sin \varphi-g \cos \varphi)$ as follows:

$$
\begin{aligned}
& 2 u_{k \varphi} u_{k x_{1}}(K \cos \varphi+g \sin \varphi) \geq-\varepsilon u_{k x_{1}}^{2}-\varepsilon^{-1}(K \cos \varphi+g \sin \varphi)^{2} u_{k \varphi}^{2}, \\
& 2 u_{k \varphi} u_{k x_{2}}(K \sin \varphi-g \cos \varphi) \geq-\varepsilon u_{k x_{2}}^{2}-\varepsilon^{-1}(K \sin \varphi-g \cos \varphi)^{2} u_{k \varphi}^{2},
\end{aligned}
$$

where $0<\varepsilon<1$, and we obtain

$$
\begin{aligned}
J\left(\nabla u_{k}\right) \geq & u_{k x_{1}}^{2}+u_{k x_{2}}^{2}-\varepsilon u_{k x_{1}}^{2}-\varepsilon u_{k x_{2}}^{2}-\varepsilon^{-1}(K \cos \varphi+g \sin \varphi)^{2} u_{k \varphi}^{2} \\
& -\varepsilon^{-1}(K \sin \varphi-g \cos \varphi)^{2} u_{k \varphi}^{2}+\left(g_{x_{1}} \cos \varphi+g_{x_{2}} \sin \varphi\right. \\
& \left.+g_{\varphi} K-K_{x_{1}} \sin \varphi+K_{x_{2}} \cos \varphi-g K_{\varphi}\right) u_{k \varphi}^{2} \\
= & (1-\varepsilon)\left(u_{k x_{1}}^{2}+u_{k x_{2}}^{2}\right)+\left(g_{x_{1}} \cos \varphi+g_{x_{2}} \sin \varphi+g_{\varphi} K\right. \\
& \left.-K_{x_{1}} \sin \varphi+K_{x_{2}} \cos \varphi-g K_{\varphi}-\varepsilon^{-1}\left(K^{2}+g^{2}\right)\right) u_{k \varphi}^{2} .
\end{aligned}
$$

Moreover, whenever (6) holds, for sufficiently close value of $\varepsilon$ to 1 , there exists an $\alpha \in \mathbb{R}$ such that $g_{x_{1}} \cos \varphi+g_{x_{2}} \sin \varphi+g_{\varphi} K-K_{x_{1}} \sin \varphi+K_{x_{2}} \cos \varphi-K_{\varphi} g-\varepsilon^{-1}\left(K^{2}+g^{2}\right) \geq \alpha>0$ in $\Omega$, and we obtain

$$
J\left(\nabla u_{k}\right) \geq(1-\varepsilon)\left|\nabla_{x} u_{k}\right|^{2}+\alpha u_{k \varphi}^{2} \geq \beta\left|\nabla_{x, \varphi} u_{k}\right|^{2},
$$

where $\beta=\min \{(1-\varepsilon), \alpha\}$.

Since the domain $D$ is bounded, $u_{k}=0$ on $\partial D \times(0,2 \pi)$ and $J\left(\nabla u_{k}\right)$ is positive definite, we have

$$
\left\|u_{k}\right\|_{L_{2}(\Omega)}^{2} \leq C_{0} \int_{\Omega}\left|\nabla_{x} u_{k}\right|^{2} d \Omega \leq C \int_{\Omega} J\left(\nabla u_{k}\right) d \Omega,
$$

where $C=C_{0} \beta^{-1}$ and $C_{0}>0$ depends on the Lebesgue measure of $D$ and does not depend on $k$.

Thus, since $u_{k} \in C_{\pi 0}^{3}, K$ and $g$ are $2 \pi$-periodic with respect to $\varphi$, after integrating (13) over $\Omega$, the divergent terms disappear and we obtain

$$
2\left(A u_{k}, u_{k}\right)_{L_{2}(\Omega)}=\int_{\Omega} J\left(\nabla u_{k}\right) d \Omega
$$

Since $u \in \Gamma(A)$, from (15) we have

$$
\begin{aligned}
\|u\|_{L_{2}(\Omega)}^{2} & \leq \underline{\lim }_{k \rightarrow \infty}\left\|u_{k}\right\|_{L_{2}(\Omega)}^{2} \\
& \leq C \lim _{k \rightarrow \infty} \int_{\Omega} J\left(\nabla u_{k}\right) d \Omega=2 C \lim _{k \rightarrow \infty}\left(A u_{k}, u_{k}\right)_{L_{2}(\Omega)}=0,
\end{aligned}
$$

which implies that $u=0$, and since $F=0$, from (5) we get $\lambda=0$. So, the uniqueness part of the proof is completed.

Now we will prove that there exists a solution $(u, \lambda)$ of Problem 3 in $\left(\Gamma(A) \cap \stackrel{\circ}{H}_{1}^{\pi}(\Omega)\right) \times$ $L_{2}(\Omega)$ by means of the following auxiliary problem. 
Determine u defined in $\Omega$ that satisfies

$$
\begin{aligned}
& A u=\mathcal{F}, \\
& \left.u\right|_{\partial D \times(0,2 \pi)}=0, \quad u(x, 0)=u(x, 2 \pi),
\end{aligned}
$$

where $\mathcal{F}=\hat{L} F$.

The solution $u$ of problem (17)-(18) will be approximated by

$$
u_{N}=\sum_{i=1}^{N} \alpha_{N_{i}} w_{i}(x, \varphi) ; \quad \alpha_{N}=\left(\alpha_{N_{1}}, \alpha_{N_{2}}, \ldots, \alpha_{N_{N}}\right) \in \mathbb{R}^{N}
$$

construction of which is based on finding the vector $\alpha_{N}$ from the system of linear algebraic equations

$$
\left(A u_{N}, w_{j}\right)_{L_{2}(\Omega)}=\left(\mathcal{F}, w_{j}\right)_{L_{2}(\Omega)}, \quad j=1,2, \ldots, N
$$

where the system of functions $\left\{w_{j}\right\}$ is taken as indicated in Section 2.3 and $u_{N}=0$ on $\partial D \times(0,2 \pi)$.

We must show that the solution of system (19) exists and is unique for any $F \in H_{2}^{\pi}(\Omega)$. To demonstrate this, let us assume that the homogeneous version of (19), i.e., the system

$$
\left(A u_{N}, w_{j}\right)_{L_{2}(\Omega)}=0, \quad j=1,2, \ldots, N
$$

has a nonzero solution $\bar{\alpha}_{N}=\left(\bar{\alpha}_{N_{1}}, \bar{\alpha}_{N_{2}}, \ldots, \bar{\alpha}_{N_{N}}\right)$. Substituting $\bar{\alpha}_{N}$ for $\alpha_{N}$, multiplying the $j$ th equation of the above homogenous system by $2 \bar{\alpha}_{N_{j}}$ and summing with respect to $j$ from 1 to $N$, we obtain

$$
2\left(A \bar{u}_{N}, \bar{u}_{N}\right)_{L_{2}(\Omega)}=0
$$

where $\bar{u}_{N}=\sum_{i=1}^{N} \bar{\alpha}_{N_{i}} w_{i}$. So, from (15) and (20) we obtain

$$
2\left(A \bar{u}_{N}, \bar{u}_{N}\right)_{L_{2}(\Omega)}=\int_{\Omega} J\left(\nabla \bar{u}_{N}\right) d \Omega=0
$$

and since the quadratic form $J\left(\nabla \bar{u}_{N}\right)$ defined in (14) is positive definite and $\bar{u}_{N}=0$ on $\partial D \times(0,2 \pi)$, we have $\bar{u}_{N}=0$ in $\Omega$. But $\left\{w_{i}\right\}$ is linearly independent and this implies that $\bar{\alpha}_{N_{i}}=0, i=1,2, \ldots, N$, which contradicts with the assumption $\bar{\alpha}_{N} \neq 0$. So, it is shown that system (19) has a unique solution $\alpha_{N}$ for any $F \in H_{2}^{\pi}(\Omega)$.

Now we estimate the solution $u_{N}$ of system (19) in terms of $F$. For this purpose, we multiply both sides of the $j$ th equation of (19) by $2 \alpha_{N_{j}}$ and sum the obtained equations with respect to $j$ from 1 to $N$ to obtain

$$
2\left(A u_{N}, u_{N}\right)_{L_{2}(\Omega)}=2\left(\mathcal{F}, u_{N}\right)_{L_{2}(\Omega)}=2\left(\hat{L} F, u_{N}\right)_{L_{2}(\Omega)} .
$$

Since $u_{N} \in C_{\pi 0}^{3}$, applying integration by parts, the right-hand side of (21) can be estimated as

$$
2\left|\left(\hat{L} F, u_{N}\right)_{L_{2}(\Omega)}\right| \leq \gamma \int_{\Omega} F_{\varphi}^{2} d \Omega+\gamma^{-1} \int_{\Omega}\left(\left(\frac{\partial}{\partial l}\right)^{*} u_{N}\right)^{2} d \Omega
$$


for $\gamma>0$, and from (15) and (21) we have

$$
\int_{\Omega} J\left(\nabla u_{N}\right) d \Omega \leq \gamma \int_{\Omega} F_{\varphi}^{2} d \Omega+\gamma^{-1} \int_{\Omega}\left(\left(\frac{\partial}{\partial l}\right)^{*} u_{N}\right)^{2} d \Omega
$$

It can be verified that for sufficiently large $\gamma>0$, from (22) we obtain

$$
\left\|u_{N}\right\|_{H_{1}^{\pi}(\Omega)} \leq C\left\|F_{\varphi}\right\|_{L_{2}(\Omega)},
$$

where the constant $C$ is independent of $N$. This implies that $\left\{u_{N}\right\}_{N=1}^{\infty}$ is bounded in $L_{2}(\Omega)$ and $\stackrel{\circ}{H}_{1}^{\pi}(\Omega)$, and since $L_{2}(\Omega)$ and $\stackrel{H}{1}_{1}^{\pi}(\Omega)$ are Hilbert spaces, it is weakly compact in $L_{2}(\Omega)$ and $\stackrel{\circ}{1}_{1}^{\pi}(\Omega)$. Therefore, there exists a subsequence, which we again denote by $\left\{u_{N}\right\}$, such that $u_{N} \rightarrow u$ weakly in $L_{2}(\Omega)$ and $\stackrel{\circ}{H}_{1}^{\pi}(\Omega)$ as $N \rightarrow \infty$ and

$$
\|u\|_{H_{1}^{\pi}(\Omega)} \leq C\left\|F_{\varphi}\right\|_{L_{2}(\Omega)}
$$

holds. Since $\left.u_{N}\right|_{\partial D \times(0,2 \pi)}=0$ and $u_{N} \rightarrow u$ weakly in $\stackrel{\circ}{H}_{1}^{\pi}(\Omega)$, we have $\left.u\right|_{\partial D \times(0,2 \pi)}=0$. From (23) we have also that $\left\{u_{N x_{1}}\right\}_{N=1}^{\infty},\left\{u_{N x_{2}}\right\}_{N=1}^{\infty}$ and $\left\{u_{N \varphi}\right\}_{N=1}^{\infty}$ are bounded and there exists a subsequence of $\left\{u_{N}\right\}$, which is again denoted by $\left\{u_{N}\right\}$, such that $u_{N x_{1}}, u_{N x_{2}}$ and $u_{N \varphi}$ converge weakly in $L_{2}(\Omega)$ to $u_{x_{1}}, u_{x_{2}}$ and $u_{\varphi}$, respectively. Taking into account that $u_{N}, w_{j} \in C_{\pi 0}^{3}, F \in H_{2}^{\pi}(\Omega)$ and applying integration by parts in (19), for $N \geq j$, we obtain

$$
\left(L u_{N}-F,(\hat{L})^{*} w_{j}\right)_{L_{2}(\Omega)}=0 .
$$

Since the linear span of $\left\{w_{j}\right\}$ is dense on the space $\stackrel{\circ}{1,2}_{1}^{\pi}(\Omega)$, passing to the limit as $N \rightarrow \infty$, we get

$$
\left(L u-F,(\hat{L})^{*} \eta\right)_{L_{2}(\Omega)}=0
$$

for every $\eta \in \stackrel{\circ}{H}_{1,2}^{\pi}(\Omega)$. If we set $\lambda=L u-F$, since $C_{0}^{\infty}(\Omega) \subset \stackrel{\circ}{H}_{1,2}^{\pi}(\Omega)$, from (24) we have $\hat{L} \lambda=0$ in the generalized functions sense. Moreover,

$$
\|\lambda\|_{L_{2}(\Omega)} \leq C\|u\|_{H_{1}^{\pi}(\Omega)}+\|F\|_{L_{2}(\Omega)}
$$

holds and, by using $\|u\|_{\hat{H}_{1}^{\pi}(\Omega)} \leq C\left\|F_{\varphi}\right\|_{L_{2}(\Omega)}$, it can be seen that (7) holds. In the above expressions, by $C$ we denote generic constants which depend only on the given functions and Lebesgue measure of the domain $D$.

Now it remains to show that $u \in \Gamma(A)$. Since $u \in L_{2}(\Omega)$ and $F \in H_{2}^{\pi}(\Omega)$, for $\mathcal{F}=\hat{L} F \in$ $L_{2}(\Omega)$, from (24) we have

$$
\left(u, A^{*} \eta\right)_{L_{2}(\Omega)}=\left(u, L^{*}(\hat{L})^{*} \eta\right)_{L_{2}(\Omega)}=\left(L u,(\hat{L})^{*} \eta\right)_{L_{2}(\Omega)}=\left(F,(\hat{L})^{*} \eta\right)_{L_{2}(\Omega)}=(\mathcal{F}, \eta)_{L_{2}(\Omega)}
$$

for any $\eta \in C_{0}^{\infty}(\Omega)$, which implies that $\mathcal{F}=A u$ in the generalized functions sense, i.e., $u \in \Gamma^{\prime \prime}(A)$.

Moreover, from (19) we have $\mathcal{P}_{N} A u_{N}=\mathcal{P}_{N} \mathcal{F}$, where $\mathcal{P}_{N}$ is the orthogonal projector. Since the system $\left\{w_{1}, w_{2}, \ldots\right\}$ is orthogonal and complete in $L_{2}(\Omega), \mathcal{P}_{N} \mathcal{F} \rightarrow \mathcal{F}$ strongly, 
i.e., $\mathcal{P}_{N} A u_{N} \rightarrow \mathcal{F}=A u$ strongly in $L_{2}(\Omega)$ as $N \rightarrow \infty$. Then, since $u_{N} \rightarrow u$ weakly in $L_{2}(\Omega)$ as $N \rightarrow \infty$, we have $\left(\mathcal{P}_{N} A u_{N}, u_{N}\right)_{L_{2}(\Omega)} \rightarrow(A u, u)_{L_{2}(\Omega)}$ as $N \rightarrow \infty$. On the other hand, since the projection operator $\mathcal{P}_{N}$ is self-adjoint in $L_{2}(\Omega)$,

$$
\left(\mathcal{P}_{N} A u_{N}, u_{N}\right)_{L_{2}(\Omega)}=\left(A u_{N}, \mathcal{P}_{N}^{*} u_{N}\right)_{L_{2}(\Omega)}=\left(A u_{N}, \mathcal{P}_{N} u_{N}\right)_{L_{2}(\Omega)}=\left(A u_{N}, u_{N}\right)_{L_{2}(\Omega)},
$$

thus $\left(A u_{N}, u_{N}\right)_{L_{2}(\Omega)} \rightarrow(A u, u)_{L_{2}(\Omega)}$ as $N \rightarrow \infty$, and so $u \in \Gamma(A)$. The proof of Theorem 1 is complete.

Proof of Lemma 1 Since $K=\frac{1}{r}$ is constant, and hence

$$
K_{x_{1}}=K_{x_{2}}=K_{\varphi}=0,
$$

to prove that condition (6) holds, we only need to show that $g$ satisfies

$$
g_{x_{1}} \cos \varphi+g_{x_{2}} \sin \varphi+g_{\varphi} \frac{1}{r}>\frac{1}{r^{2}}+g^{2}
$$

By taking into account that $M=\sup _{\left(x_{1}, x_{2}\right) \in D}\left(x_{1}^{2}+x_{2}^{2}\right)$ and $r>3 \sqrt{M}$, for the function

$$
g\left(x_{1}, x_{2}, \varphi\right)=\frac{1}{3 M}\left(x_{1} \cos \varphi+x_{2} \sin \varphi\right)
$$

given in (8) defined on $D \times(0,2 \pi)$, we obtain

$$
\begin{aligned}
& g_{x_{1}} \cos \varphi+g_{x_{2}} \sin \varphi+g_{\varphi} \frac{1}{r}-\frac{1}{r^{2}}-g^{2} \\
&=\frac{1}{3 M}\left(1+\frac{1}{r}\left(-x_{1} \sin \varphi+x_{2} \cos \varphi\right)\right) \\
& \quad-\frac{1}{r^{2}}-\frac{1}{9 M^{2}}\left(x_{1} \cos \varphi+x_{2} \sin \varphi\right)^{2} \\
& \geq \frac{1}{3 M}\left(1-\frac{1}{r} \sqrt{M}\right)-\frac{1}{r^{2}}-\frac{1}{9 M} \\
&> \frac{1}{3 M}\left(1-\frac{1}{3}\right)-\frac{2}{9 M} \\
&= 0,
\end{aligned}
$$

and the proof is complete.

Proof of Lemma 2 For

$$
\begin{aligned}
K\left(x_{1}, x_{2}, \varphi\right) & =\left(x_{1} \sin \varphi-x_{2} \cos \varphi+\left(R^{2}-\left(x_{1} \cos \varphi+x_{2} \sin \varphi\right)^{2}\right)^{1 / 2}\right)^{-1} \\
& =\frac{\left(R^{2}-\left(x_{1} \cos \varphi+x_{2} \sin \varphi\right)^{2}\right)^{1 / 2}-x_{1} \sin \varphi+x_{2} \cos \varphi}{R^{2}-x_{1}^{2}-x_{2}^{2}},
\end{aligned}
$$


we have

$$
\begin{aligned}
K_{x_{1}}= & \frac{1}{\left(R^{2}-x_{1}^{2}-x_{2}^{2}\right)^{2}}\left(\left(\frac{-\left(x_{1} \cos \varphi+x_{2} \sin \varphi\right) \cos \varphi}{\left(R^{2}-\left(x_{1} \cos \varphi+x_{2} \sin \varphi\right)^{2}\right)^{1 / 2}}-\sin \varphi\right)\left(R^{2}-x_{1}^{2}-x_{2}^{2}\right)\right. \\
& \left.+2 x_{1}\left(\left(R^{2}-\left(x_{1} \cos \varphi+x_{2} \sin \varphi\right)^{2}\right)^{1 / 2}-x_{1} \sin \varphi+x_{2} \cos \varphi\right)\right), \\
K_{x_{2}}= & \frac{1}{\left(R^{2}-x_{1}^{2}-x_{2}^{2}\right)^{2}}\left(\left(\frac{-\left(x_{1} \cos \varphi+x_{2} \sin \varphi\right) \sin \varphi}{\left(R^{2}-\left(x_{1} \cos \varphi+x_{2} \sin \varphi\right)^{2}\right)^{1 / 2}}+\cos \varphi\right)\left(R^{2}-x_{1}^{2}-x_{2}^{2}\right)\right. \\
& \left.+2 x_{2}\left(\left(R^{2}-\left(x_{1} \cos \varphi+x_{2} \sin \varphi\right)^{2}\right)^{1 / 2}-x_{1} \sin \varphi+x_{2} \cos \varphi\right)\right), \\
K_{\varphi}= & \frac{-\left(x_{1} \cos \varphi+x_{2} \sin \varphi\right)}{\left(R^{2}-x_{1}^{2}-x_{2}^{2}\right)}\left(\frac{\left(-x_{1} \sin \varphi+x_{2} \cos \varphi\right)}{\left(R^{2}-\left(x_{1} \cos \varphi+x_{2} \sin \varphi\right)^{2}\right)^{1 / 2}}+1\right)
\end{aligned}
$$

and

$$
\begin{aligned}
- & K_{x_{1}} \sin \varphi+K_{x_{2}} \cos \varphi \\
= & \frac{1}{\left(R^{2}-x_{1}^{2}-x_{2}^{2}\right)^{2}}\left(R^{2}-\left(x_{1} \cos \varphi+x_{2} \sin \varphi\right)^{2}+\left(x_{1} \sin \varphi-x_{2} \cos \varphi\right)^{2}\right. \\
& \left.-2\left(x_{1} \sin \varphi-x_{2} \cos \varphi\right)\left(R^{2}-\left(x_{1} \cos \varphi+x_{2} \sin \varphi\right)^{2}\right)^{1 / 2}\right) \\
= & K^{2} .
\end{aligned}
$$

Since $M=\sup _{\left(x_{1}, x_{2}\right) \in D}\left(x_{1}^{2}+x_{2}^{2}\right)$ and $R>\sqrt{M}$, for

$$
g\left(x_{1}, x_{2}, \varphi\right)=\frac{1}{2 M}\left(x_{1} \cos \varphi+x_{2} \sin \varphi\right)
$$

given in (12) defined on $D \times(0,2 \pi)$, we have

$$
\begin{aligned}
-K_{\varphi} g= & \frac{\left(x_{1} \cos \varphi+x_{2} \sin \varphi\right)}{\left(R^{2}-x_{1}^{2}-x_{2}^{2}\right)}\left(\frac{\left(-x_{1} \sin \varphi+x_{2} \cos \varphi\right)}{\left(R^{2}-\left(x_{1} \cos \varphi+x_{2} \sin \varphi\right)^{2}\right)^{1 / 2}}+1\right) \\
& \times \frac{1}{2 M}\left(x_{1} \cos \varphi+x_{2} \sin \varphi\right) \\
= & \frac{\left(x_{1} \cos \varphi+x_{2} \sin \varphi\right)^{2}\left(-x_{1} \sin \varphi+x_{2} \cos \varphi+\left(R^{2}-\left(x_{1} \cos \varphi+x_{2} \sin \varphi\right)^{2}\right)^{1 / 2}\right)}{2 M\left(R^{2}-x_{1}^{2}-x_{2}^{2}\right)\left(R^{2}-\left(x_{1} \cos \varphi+x_{2} \sin \varphi\right)^{2}\right)^{1 / 2}} \\
\geq & 0 .
\end{aligned}
$$

Hence, to prove that condition (6) holds, we only need to show that

$$
g_{x_{1}} \cos \varphi+g_{x_{2}} \sin \varphi+g_{\varphi} K>g^{2}
$$

holds. If we take into account again that $M=\sup _{\left(x_{1}, x_{2}\right) \in D}\left(x_{1}^{2}+x_{2}^{2}\right)$ and $R>\sqrt{M}$, then we obtain

$$
\begin{aligned}
& g_{x_{1}} \cos \varphi+g_{x_{2}} \sin \varphi+g_{\varphi} K-g^{2} \\
& \quad=\frac{1}{2 M}\left(1+\frac{-x_{1} \sin \varphi+x_{2} \cos \varphi}{x_{1} \sin \varphi-x_{2} \cos \varphi+\left(R^{2}-\left(x_{1} \cos \varphi+x_{2} \sin \varphi\right)^{2}\right)^{1 / 2}}\right)
\end{aligned}
$$




$$
\begin{aligned}
& -\frac{1}{4 M^{2}}\left(x_{1} \cos \varphi+x_{2} \sin \varphi\right)^{2} \\
\geq & \frac{1}{2 M} \frac{\left(R^{2}-\left(x_{1} \cos \varphi+x_{2} \sin \varphi\right)^{2}\right)^{1 / 2}}{x_{1} \sin \varphi-x_{2} \cos \varphi+\left(R^{2}-\left(x_{1} \cos \varphi+x_{2} \sin \varphi\right)^{2}\right)^{1 / 2}}-\frac{1}{4 M}
\end{aligned}
$$

$>0$.

\section{The proof is complete.}

\section{Competing interests}

The author declares that he has no competing interests.

\section{Received: 2 August 2013 Accepted: 19 August 2013 Published: 8 September 2013}

\section{References}

1. Isakov, V: Inverse Problems for Partial Differential Equations. Springer, New York (2006)

2. Radon, J: Über die Bestimmung von Funktionen durch ihre Integralwerte längs gewisser Mannigfaltigkeiten. Ber. Verh. Sächs. Akad. Wiss. Leipz., Math.-Nat.wiss. Kl. 69, 262-277 (1917)

3. Natterer, F, Wübbeling, F: Mathematical Methods in Image Reconstruction. Monographs on Mathematical Modeling and Computation, vol. 5. SIAM, Philadelphia (2001)

4. Natterer, F: The Mathematics of Computerized Tomography. Vieweg+Teubner, Wiesbaden (1986)

5. Cormack, AM: Representation of a function by its line integrals, with some radiological applications. J. Appl. Phys. 34, 2722-2727 (1963)

6. Cormack, AM: The Radon transform on a family of curves in the plane. Proc. Am. Math. Soc. 83, 325-330 (1981)

7. Natterer, F: Inversion of attenuated Radon transform. Inverse Probl. 17, 113-119 (2001)

8. Novikov, RG: An inversion formula for the attenuated X-ray transformation. Ark. Mat. 40(1), 145-167 (2002)

9. Quinto, ET: Radon transforms on curves in the plane. In: Tomography, Impedance Imaging and Integral Geometry. Lectures in Applied Mathematics, vol. 30, pp. 231-244. Am. Math. Soc., Providence (1994)

10. Ambartsoumian, G, Gouia-Zarrad, R, Lewis, M: Inversion of the circular Radon transform on an annulus. Inverse Probl. $26,105015(2010)$

11. Nguyen, MK, Truong, TT: Inversion of a new circular-arc Radon transform for Compton scattering tomography. Inverse Probl. 26, 065005 (2010)

12. Rigaud, G, Nguyen, MK, Louis, AK: Novel numerical inversions of two circular-arc Radon transforms in Compton scattering tomography. Inverse Probl. Sci. Eng. 20(6), 809-839 (2012)

13. Amirov, AK: Integral Geometry and Inverse Problems for Kinetic Equations. VSP, Utrecht (2001)

14. Gelfand, IM, Gindikin, SG, Graev, MI: Selected Topics in Integral Geometry. Am. Math. Soc., Providence (2003)

15. Romanov, VG: Integral Geometry and Inverse Problems for Hyperbolic Equations. Springer, Berlin (1974)

16. Sharafutdinov, VA: Integral Geometry of Tensor Fields. VSP, Utrecht (1994)

17. Arridge, SR: Optical tomography in medical imaging. Inverse Probl. 15, R41-R93 (1999)

18. Bal, G: Inverse transport theory and applications. Inverse Probl. 25, 053001 (2009)

19. Stefanov, P: Inverse problems in transport theory. In: Uhlmann, G (ed.) Inside Out: Inverse Problems and Applications. MSRI Publications, vol. 47. Cambridge University Press, Cambridge (2003)

20. Tamasan, A: An inverse boundary value problem in two-dimensional transport. Inverse Probl. 18, 209-219 (2002)

21. Anikonov, DS, Kovtanyuk, AE, Prokhorov, IV: Transport Equation and Tomography. VSP, Utrecht (2002)

22. Case, KM, Zweifel, PF: Linear Transport Theory. Addison-Wesley, Reading (1967)

23. Klibanov, MV, Yamamoto, M: Exact controllability for the time dependent transport equation. SIAM J. Control Optim. 46(6), 2071-2095 (2007)

24. Amirov, AK: Existence and uniqueness theorems for the solution of an inverse problem for the transport equation. Sib. Math. J. 27, 785-800 (1986)

25. Amirov, A, Yildiz, M, Ustaoglu, Z: Solvability of a problem of integral geometry via an inverse problem for a transport-like equation and a numerical method. Inverse Probl. 25, 095002 (2009)

26. Ustaoglu, Z, Heydarov, B, Amirov, S: On the solvability and approximate solution of a two dimensional coefficient inverse problem for a transport-like equation. Inverse Probl. 26, 115019 (2010)

27. Amirov, A, Ustaoglu, Z, Heydarov, B: Solvability of a two dimensional coefficient inverse problem for transport equation and a numerical method. Transp. Theory Stat. Phys. 40(1), 1-22 (2011)

28. Golgeleyen, I: An integral geometry problem along geodesics and a computational approach. An. Univ. "Ovidius" Constanţa, Ser. Mat. 18(2), 91-112 (2010)

29. Golgeleyen, I: An inverse problem for a generalized transport equation in polar coordinates and numerical applications. Inverse Probl. 29, 095006 (2013)

30. Lavrent'ev, MM, Anikonov, YE: A certain class of problems in integral geometry. Sov. Math. Dokl. 8, 1240-1241 (1967)

31. Klibanov, MV, Timonov, A: Carleman Estimates for Coefficient Inverse Problems and Numerical Applications. VSP, Utrecht (2004)

32. Courant, R, Hilbert, D: Methods of Mathematical Physics. Partial Differential Equations, vol. 2. Interscience, New York (1962)

33. Lavrent'ev, MM, Romanov, VG, Shishatskii, SP: III-Posed Problems of Mathematical Physics and Analysis. Am. Math. Soc. Providence (1986)

34. Mikhailov, VP: Partial Differential Equations. Mir, Moscow (1978)

35. Evans, LC: Partial Differential Equations. Am. Math. Soc., Providence (1998)

36. Lions, JL, Magenes, E: Nonhomogeneous Boundary Value Problems and Applications. Springer, London (1972) 
doi:10.1186/1687-2770-2013-202

Cite this article as: Ustaoglu: A generalization on the solvability of integral geometry problems along plane curves. Boundary Value Problems 2013 2013:202.

Submit your manuscript to a SpringerOpen ${ }^{\circ}$ journal and benefit from:

- Convenient online submission

- Rigorous peer review

- Immediate publication on acceptance

Open access: articles freely available online

- High visibility within the field

- Retaining the copyright to your article

Submit your next manuscript at $\boldsymbol{s p r i n g e r o p e n . c o m ~}$ 